\title{
Minimal access tricuspid valve surgery
}

\author{
Joseph Lamelas \\ Division of Cardiovascular Surgery, Michael E. DeBakey Department of Surgery, Houston, TX, USA \\ Correspondence to: Joseph Lamelas, MD. Associate Chief of Cardiac Surgery, Baylor St. Luke's Medical Center, Texas Heart Institute, One Baylor \\ Plaza, MS: BCM 390, Houston, Texas 77030, USA. Email: jlamelasmd@aol.com.
}

Submitted Sep 22, 2016. Accepted for publication Feb 16, 2017.

doi: 10.21037/acs.2017.03.17

View this article at: http://dx.doi.org/10.21037/acs.2017.03.17

\section{Clinical vignette}

The minimally invasive, right mini-thoracotomy technique described can be applied to patients that require a concomitant tricuspid valve repair or replacement at the time of a mitral valve procedure. This has been the most common scenario where tricuspid valve surgery is performed. This approach is applicable to patients requiring isolated tricuspid valve surgery. In addition, patients who have had prior cardiac surgery presenting with tricuspid valve disease are candidates for a lesser invasive approach. All patients with primary or late secondary tricuspid regurgitation (TR) are considered candidates unless arterial cannulation is not possible.

\section{Surgical techniques}

\section{Preparation}

All patients undergo a pre-operative cardiac catheterization and echocardiogram. A computed tomography (CT) angiogram of the chest, abdomen and pelvis to evaluate for aortic and peripheral vascular disease is not routinely performed. We have demonstrated extremely low stroke rates with this approach. The patient is intubated with a single lumen endotracheal tube. Double lumen endotracheal tubes or bronchial blockers are not utilized and single lung ventilation is avoided. A Swan Ganz catheter and transesophageal echocardiogram probe are also inserted. A through echocardiographic evaluation is performed prior to positioning the patient.

\section{Exposition}

All patients are placed in the supine position during anesthetic induction. Thereafter, a roll is placed behind the scapula. In male patients, the right arm is positioned slightly off the right side of the table. In female patients, the patient is rolled to the left and the right arm is placed over the head. The hips are positioned as supine as possible on the operating table to facilitate peripheral cannulation.

The fourth or fifth intercostal space provides optimal exposure of both the mitral and tricuspid valves. The middle point of the entire sternum is an excellent external landmark that usually correlates with this interspace as well as the location of the mitral valve. In male patients, a $5-6 \mathrm{~cm}$ incision is performed lateral to the anterior axillary line and starting at the point which correlates to the middle of the sternum. In female patients, with the arm placed over the head, the incision is made in the lateral mammary crease and extends equidistant from the point that correlates to the middle of the sternum.

\section{Operation}

A femoral platform is preferred to establish cardiopulmonary bypass. The femoral artery is cannulated with a 15-19 French Biomedicus arterial cannula. The size will be dependent upon the patients' body surface area. If severe peripheral vascular disease is present, axillary artery or central cannulation is performed. The femoral vein is cannulated with a 25 French Biomedicus venous cannula in all patients utilizing transesophageal echocardiogram guidance. Percutaneous internal jugular cannulation is no longer utilized in an attempt to simplify the procedure as well as limit the invasiveness.

Cardiopulmonary bypass is initiated using a closed membrane oxygenator, and a roller pump. Venous drainage is augmented with vacuum assistance applying negative 
pressures of 35 to $65 \mathrm{mmHg}$ as needed to decompress the right heart. This has allowed adequate venous drainage. The patients' temperature is allowed to drift to 32 degrees.

In cases where the mitral valve needs to be addressed, trans-incisional direct aortic cross clamping is performed utilizing a flexible and retractable shaft cross clamp. An angio-catheter placed directly through the incision is utilized to deliver an extended effect cardioplegia solution directly into the aortic root. We have recently converted to a modified Del Nido cardioplegia solution consisting of 4 parts blood and one part crystalloid solution containing $40 \mathrm{mEq}$ of potassium per liter. A two liter induction dose allows for at least 90 minutes of safe myocardial protection. If required an additional $400 \mathrm{cc}$ of cardioplegia is administered.

Thereafter a left atriotomy is initially performed and a sump suction is placed in the left inferior pulmonary vein. The valve replacement and/or repair is carried out under direct vision utilizing standard techniques and long shafted instruments. Carbon dioxide is infused into the operative field during the entire procedure. When completed, the left atriotomy is partially closed, leaving a sump suction in the left atrium. The superior and inferior cava are encircled with rubber vessel loops. The long femoral venous cannula is withdrawn into the infra- diaphragmatic inferior vena cava and the cava is snared. The superior vena cava is simultaneously snared. The right atriotomy is performed and a second sump suction which has been tunneled through the chest tube incision is placed through the right atriotomy and into the superior vena cava. Of note, the superior vena cava snare needs to be temporarily released during this maneuver. The operative field will be briefly obscured with blood. This does not need to be performed in a rushed fashion since a few minutes of inflow occlusion of the cava is well tolerated. An alternative approach would be to insert the sump suction through a separate stab wound in the superior vena cava prior to performing the right atriotomy. Thereafter, repair techniques on the tricuspid valve can be accomplished. The Swan Ganz catheter is left in place during the repair. Once completed, the right atriotomy is closed in a two-layered fashion, with the first being a running suture line and the second a mattress closure. The sump suction is removed after completing the first suture line and the long femoral venous cannula is advanced into the right atrium. Of note, in patients requiring re-operative tricuspid surgery, the cavas are not snared and the long venous cannula is not withdrawn. The right atriotomy is performed directly through the adherent pericardium. Although mild to moderate air return will be noted in the venous cannula during the tricuspid procedure, this is well tolerated.

Alternatively, if an isolated tricuspid valve repair is performed, the heart does not need to be arrested and the procedure can be performed with the heart beating. With this approach, excessive blood return from the coronary sinus can limit visibility of the operative field and an additional sump suction needs to be placed in the right atrium.

\section{Completion}

In those patients where the heart is arrested, the patient is placed in a Trendelenburg position and once the cross-clamp is removed, an angio-catheter inserted in the aortic root aids with removal of air. One Blake chest tube is positioned in the pleural cavity and another in the pericardial sac through the oblique sinus. These, along with the temporary ventricular pacing wire and the On-Q pain relief system are passed through the chest tube incision. A number 2 suture is then placed in a figure of eight fashion to approximate the ribs.

Patients requiring re-operative surgery on the mitral and/or tricuspid valves are optimal candidates for a lesser invasive, right thoracotomy approach. Pleural adhesions are rarely encountered if the first operation was performed by a sternotomy. Patients who have had a prior right thoracotomy are candidates although adhesions of the lung to the chest wall can be challenging. In these cases, a bronchial blocker will be used to isolate the right lung during the entry and dissection.

\section{Clinical results}

When compared to a standard median sternotomy approach, the potential benefits of minimally invasive valve surgery include: reduced surgical trauma, blood loss, re-operation for bleeding, and pain; a shorter intensive care unit and hospital length of stay, as well as a more rapid return to functional activity (1-5). Isolated tricuspid valve repair has been associated with mortality rates of $8.2-9.5 \%(6,7)$. Mortality rates with re-operative isolated tricuspid valve surgery have ranged from 13.2-37\% (8-10). Approximately half of all patients undergoing isolated tricuspid surgery have a history of prior cardiac surgery.

\section{Isolated TVRs (primary and redo)}

A total of 2,945 heart operations performed at the 
Mount Sinai Medical Center, Miami Beach by the author between January 2009 and April 2013 were retrospectively reviewed to identify patients who had undergone isolated, minimally invasive tricuspid valve surgery via a right minithoracotomy approach. Details of operative times, intensive care unit (ICU) and hospital lengths of stay, postoperative complications, and mortality were analyzed.

A total of 12 patients (eight females, four males; mean age $68 \pm 18$ years) were identified. The median LVEF, pulmonary artery systolic pressure, and preoperative serum creatinine level were 58\% (IQR 47-64\%), $40 \mathrm{mmHg}$ (IQR 31-45 mmHg), and $0.9 \mathrm{mg} / \mathrm{dl}$ (IQR $0.8-1.1 \mathrm{mg} / \mathrm{dL}$ ), respectively. Comorbidities included hypertension (83\%), chronic obstructive pulmonary disease (42\%) and diabetes mellitus (17\%).

All patients had severe TR, with the etiology of tricuspid valve disease being functional in seven cases, pacemaker device-induced in three, rheumatic in one case, and flail anterior leaflet in one case. Six patients $(50 \%)$ underwent reoperation with a history of previous coronary artery bypass graft surgery $(n=2)$ or valve surgery $(n=4)$. There was no statistically significant difference between patients who underwent first-time surgery and those who had a reoperation. Aortic cross-clamping was required in two patients, and the majority (92\%) underwent tricuspid valve repair. The median $\mathrm{CPB}$ and aortic cross-clamp times were $106 \mathrm{~min}$ (IQR 82-122 $\mathrm{min}$ ) and $105 \mathrm{~min}$ (IQR 80-130 $\mathrm{min}$ ), respectively. An intraoperative packed red blood cells (PRBC) transfusion was needed in $50 \%$ of cases, with a median of 1 unit of PRBC (IQR 1-2 unit). None of the patients required conversion to full median sternotomy.

Postoperative complications included prolonged ventilation in six patients, reintubation in two, atrial fibrillation in two, and renal failure in one patient. However, there were no postoperative myocardial infarctions, cerebrovascular events, reoperations for bleeding, or deepwound infections. The median intensive care unit and hospital lengths of stay were $84 \mathrm{~h}$ (IQR 47-157 h) and 7 days (IQR 6-12 days), respectively. There were two in-hospital deaths $(17 \%)$, attributed to cardiogenic and septic shock (one each). There were no additional deaths at 30 days postoperatively. Survival at a median of 25.6 months follow up (IQR 10.4-30.3 months) was 67\% (11).

\section{Advantages}

The mini thoracotomy approach allows for excellent direct and unimpaired anatomical visualization of both the mitral and tricuspid valves as compared to a sternotomy. Avoiding a sternotomy benefits patients at an increased risk for sternal wound infections. Sternal sparring operations allow patients to unimpeded pulmonary toilet, coughing and ambulation due to superior chest wall stability. In addition, the incidence of bleeding as well as transfusion requirements are diminished because of less tissue trauma and cardiac manipulation. Recovery, both in the hospital and back to a normal lifestyle can be accelerated in this higher risk population.

\section{Caveats}

Choosing the correct interspace is crucial to obtain adequate exposure. Selecting the mid point on the sternum as a landmark for entry into the chest and performing the incision lateral to the anterior axillary line or lateral mammary crease is paramount. Despite being more lateral and further away from the heart, the superior anatomical exposure facilitates any complex replacement or reconstruction.

\section{Acknowledgements}

None.

\section{Footnote}

Conflicts of Interest: The author has no conflicts of interest to declare.

\section{References}

1. Schmitto JD, Mokashi SA, Cohn LH. Minimally-invasive valve surgery. J Am Coll Cardiol 2010;56:455-62.

2. Lamelas J, Sarria A, Santana O, et al. Outcomes of minimally invasive valve surgery versus median sternotomy in patients 75 years or greater. Ann Thorac Surg 2011;91:75-80.

3. Santana O, Reyna J, Grana R, et al. Outcomes of minimally invasive valve surgery versus standard sternotomy in obese patients undergoing isolated valve surgery. Ann Thorac Surg 2011;91:406-10.

4. Santana O, Reyna J, Benjo AM, et al. Outcomes of minimally invasive valve surgery in patients with chronic obstructive pulmonary disease. Eur J Cardio-Thoracic Surg 2012;42:648-52.

5. Santana O, Funk M, Zamora C, et al. Staged percutaneous 
coronary intervention and minimally invasive valve surgery: results of a hybrid approach to concomitant coronary and valvular disease. J Thorac Cardiovasc Surg 2012;144:634-9.

6. Vassileva CM, Shabosky J, Boley T, et al. Tricuspid valve surgery: the past 10 years from the Nationwide Inpatient Sample (NIS) database. J Thorac Cardiovasc Surg 2012;143:1043-9.

7. Kilic A, Saha-Chaudhuri P, Rankin JS, et al. The Annals of Thoracic Surgery, Trends and Outcomes of Tricuspid Valve Surgery in North America: An Analysis of More Than 50,000 Patients From The Society of Thoracic Surgeons Database, 2013;5:1546-52.

Cite this article as: Lamelas J. Minimal access tricuspid valve surgery. Ann Cardiothorac Surg 2017;6(3):283-286. doi: 10.21037/acs.2017.03.17
8. Jeganathan R, Armstrong S, Al-Alao B, et al. The risk and outcomes of reoperative tricuspid valve surgery. Ann Thorac Surg 2013;95:119-24.

9. Pfannmüller B, Moz M, Misfeld M, et al. Isolated tricuspid valve surgery in patients with previous cardiac surgery. J Thorac Cardiovasc Surg 2013;146:841-7.

10. Bernal JM, Morales D, Revuelta C, et al. Reoperations after tricuspid valve repair. J Thorac Cardiovasc Surg 2005;130:498-503.

11. Urbandt P, Santana O, Mihos CG, et al. Minimally invasive approach for isolated tricuspid valve surgery. $\mathrm{J}$ Heart Valve Dis 2014;23:783-7. 